$5 \%$ Kohlensäure. In die entsprechenden Gefäße wurde eine Fermentmenge pipettiert, deren Aktivität einer Phosphatabspaltung von $5 \mu$ Molen Phosphat pro Stde. aus FDP entsprach. Weiterhin wurde eine Einheit Insulin zum Inkubationsmedium hinzugesetzt.

Die erhaltenen Versuchsergebnisse zeigt die Abb. 1 .

Aus der Darstellung geht hervor, daß die durch FDPase hervorgerufene Hemmung der Glykolyse durch den Insulinzusatz teilweise wieder kompensiert wird, während die normale Glykolyse der Asciteszellen durch einen Insulinzusatz nur unwesentlich beeinflußt wird. Zur Klärung des Wirkungsmechanismus haben wir FDPase 20 Min. lang mit steigenden Mengen Insulin inkubiert und dann die Aktivität der FDPase bestimmt ${ }^{12}$.

Die Versuchsergebnisse sind in der Abb. 2 dargestellt.

Es zeigte sich, daß durch relativ hohe Insulinmengen eine völlige Inaktivierung des Fermentes erreicht werden kann.

Diese Resultate sprechen dafür, daß das Insulin bis zu einem gewissen Grade als Antagonist der

12 B. M. Pogell u. R. W. McGilvery, Methods in Enzymology, Am. Press. 1957, III, S. 543.

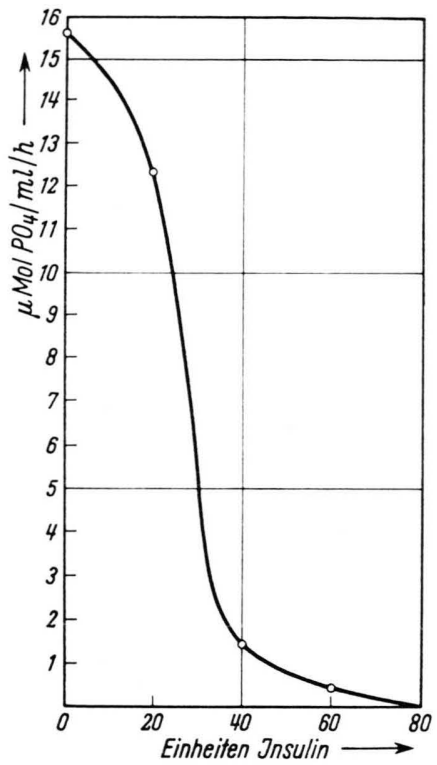

Abb. 2. Abhängigkeit der FDPase-Aktivität vom InsulinZusatz.

FDPase betrachtet werden kann. Inwieweit das Insulin selbst schon als Maskierungssubstanz auf eine aktive FDPase wirken kann, ist durch unsere Versuche noch nicht zu entscheiden. Andererseits könnte das Insulin auch über unbekannte Zwischenprodukte wirken.

\title{
Weitere Beiträge zur Kenntnis der chemischen Zusammensetzung des Giftes von Ameisen aus der Gattung Formica
}

\author{
Von M. F. H. Osman * und J. Brander ** \\ Aus dem Institut für Angewandte Zoologie und dem Chemischen Institut der Universität Würzburg \\ (Z. Naturforschg. 16 b, 749-753 [1961]; eingegangen am 16. März 1961) \\ 1. An 10 Proben von 3 Arten der Gattung Formica wurde festgestellt, daß im Gift zwischen 61 bis \\ 65\% reine Ameisensäure enthalten sind. Der Vergleich der titrimetrischen mit einer für $\mathrm{HCOOH}$ spe- \\ zifischen gravimetrischen Bestimmungsmethode läßt den Schluß zu, daß außer Ameisensäure keine \\ andere Säure in nennenswerter Menge vorliegt. \\ 2. In allen Giftproben wurden die in Aceton löslichen bzw. mit Aceton fällbaren Anteile der \\ Begleitsubstanzen getrennt bestimmt. Die Gesamtmenge der Trockensubstanzen beträgt 1,17-1,82\% \\ des Rohgiftgewichtes. \\ 3. In 8 Proben wurde die $\mathrm{NH}_{3}$-Menge, in 2 Proben nach Hydrolyse der acetonlöslichen Substanzen \\ der Gehalt an Aminosäuren bestimmt. In einer Probe überwinternder Ameisen bestehen 19,85\%, bei \\ Sommerameisen 4,83\% der acetonlöslichen Substanzen aus Ammoniak und 15-17\% aus Aminosäuren. \\ 4. Im Ameisengift ist kein Phosphat enthalten.
}

Es ist lange bekannt, daß das Gift der zur Unterfamilie der Formicinae gehörenden Ameisen einen hohen Gehalt an Ameisensäure besitzt. Die Mehrzahl der früheren Autoren schenkte dieser Säure besondere Beachtung und es wurde gezeigt, daß die insektizide und antibiotische Wirkung des Giftes im we-

Ständige Anschrift: Dept. of Entomology, Faculty of ** Ständige Anschrift: Allensbach/Bodensee, Sonnenhöhe. Science, Cairo University, Egypt. 
sentlichen der Ameisensäure zukommt ${ }^{1}$. Die chemische Analyse beschränkte sich meist auf die Bestimmung des Gehaltes an Ameisensäure in den Giften. Stumper $^{2}$ stellte fest, daß die Ameisensäure die einzige freie flüchtige Säure im Gift der Formica rufa ist und ermittelte ihren Gehalt mit $21-72$ Prozent. O'Rourke ${ }^{3}$ bestätigte colorimetrisch die Anwesenheit dieser Säure im Gift einiger Formicinae. Stumper ${ }^{4}$ bestimmte den durchschnittlichen Ameisensäuregehalt des Giftes von Formica rufa mit 51,3 $\pm 3,3 \%$, von $F$. nigricans mit $47,1 \pm 5,6$ Prozent. Bei Formica polyctena wurden von Отто ${ }^{1} 52 \%$ und $55,4 \%$ gefunden, in Übereinstimmung mit den Ergebnissen von Stumper. Beide Autoren verwendeten die gleiche Methode zur Giftgewinnung und bestimmten die Säure durch Titrieren mit $n / 100$ und $n / 10-\mathrm{NaOH}$.

In einer Arbeit über die Stoffe, welche die Ameisensäure im Gift begleiten, zeigte Stumper ${ }^{5}$, daß beim Eintrocknen an der Luft oder bei $100^{\circ} \mathrm{C} 3,6$ bis $4 \%$ Rückstand von starkem würzig-aromatischem Geruch bleiben. Davon werden ungefähr $75 \%$ einem neuen organischen Duftstoff, vermutlich einem Terpen zugeschrieben, für das der Name Formicein vorgeschlagen wurde. Beim Erhitzen des Giftes auf $900{ }^{\circ} \mathrm{C}$ wird ein brauner Rückstand von etwa $0,5 \%$ erhalten, der Eisen, Calcium und Alkalien enthält.

Die vorliegende Arbeit enthält eire quantitative, gravimetrische Bestimmung des AmeisensäureGehaltes im Gift von drei verschiedenen FormicaArten sowie einen Vergleich der titrimetrischen und der gravimetrischen Bestimmungsmethoden. Einige Bestimmungen der Begleitsubstanzen im Ameisengift, insbesondere der Anteile an in Aceton löslichen bzw. mit Aceton fällbaren Substanzen sowie des $\mathrm{NH}_{3}$ und in einzelnen Fällen der Aminosäuren nach Hydrolyse des in Aceton löslichen Anteils wurden ebenfalls durchgeführt.

\section{Versuchstiermaterial und Gewinnung des Giftes}

Insgesamt wurden 10 Proben von drei verschiedenen Formica-Arten untersucht, von denen eine im Winter (Probe Nr. 1, Tab. 1), alle anderen im Sommer entnommen wurden. Die Tab. 1 enthält alle Angaben zu

1 A. Nascimbene u. M. Pavan, Bull. Soc. Medicochirurgica di Pavia. 3-4, 16 [1950]; M. Pavan u. A. Nascimbene, Atti. Soc. ital. Sci. natur. 88, 8 [1949]; D. Oтто, Zool. Anz. 164, 42 [1960] ; M. F. H. Osman u. W. Kloft, im Druck.

2 R. Stumper, C. R. hebd. Séances Acad. Sci. 174, 66 [1922] ; 174, 413 [1922] ; Ann. Sci. natur. 1922, 105.

3 F. J. O'Rourke, Ann. ent. Soc. Amer. 43, 437 [1950].

${ }^{4}$ R. Stumper, C. R. hebd. Séances Acad. Sci. 233, 1144 den Versuchstierproben sowie über die Menge an gewonnenem Gift und die Anzahl der benötigten Arbeiterinnen.

Die Ameisen wurden mit $\mathrm{CO}_{2}$ betäubt, sodann mußten zur Freilegung der Giftblase mit einer feinen Pinzette die letzten drei Abdominaltergite entfernt werden. Mit einer sehr feinen, durch Ausziehen eines Glasrohres von $2 \mathrm{~mm}$ Durchmesser hergestellten Kapillare wurde die Giftblase angestochen, deren ganzer Inhalt sich so herausziehen läßt ${ }^{*}$. Vor dem Punktieren wurde die Oberfläche der Giftblase durch kleine Stücke Filtrierpapier sorgfältig abgetrocknet, um jede Verunreinigung des Giftes mit Haemolymphe zu vermeiden. In Mikroreagenzgläsern von $2 \mathrm{~mm}$ Durchmesser wurde das Gift von vielen Arbeiterinnen (Mengen vgl. Tab. 1) gesammelt. Während der Präparationen wurden die Reagenzgläser in Eis-Kochsalz-Mischung gekühlt, später zugeschmolzen und im Kühlschrank aufbewahrt.

$$
\text { Bestimmung der Ameisensäure }
$$

Die Ameisensäure wurde im allgemeinen titrimetrisch bestimmt, nachdem durch einen Vergleich mit der anschließend geschilderten, für Ameisensäure spezifischen gravimetrischen Methode die Brauchbarkeit der acidimetrischen Bestimmungen erwiesen worden war.

Zur Einwaage wurde jeweils eine gewisse Menge des Giftes in ein feines Tropfrohr eingesaugt, dieses auf einer Analysen-Schnellwaage gewogen, entleert, und ohne Verzug wieder gewogen. Die Einwaage ergibt sich aus der Differenz beider Wägungen.

Zur Titration wurde das Gift in wenig dest. Wasser gelöst und mit $n / 100-\mathrm{NaOH}$ titriert.

Die gravimetrische Bestimmung wurde nach $\mathrm{S}_{\mathrm{CALA}}{ }^{6}$, ${\text { Franzen und } \text { Greve }^{6}{ }^{6} \text { sowie Riesser }}^{6}$ mit reiner Ameisensäure als Testsubstanz nachgeprüft. Die Reaktion lieferte genaue Werte und ist spezifisch für Ameisensäure. Sie beruht auf der Reduktion von $\mathrm{HgCl}_{2}$ zu $\mathrm{Hg}_{2} \mathrm{Cl}_{2}$ durch Ameisensäure.

Arbeitsvorschrift: Die Einwaage wird in wenig dest. Wasser gelöst, etwas Essigsäure und $10 \mathrm{ml}$ Sublimatmischung hinzugegeben, die $200 \mathrm{~g} \mathrm{HgCl}_{2}, 300 \mathrm{~g} \mathrm{Na}$ Acetat und $80 \mathrm{~g} \mathrm{NaCl}$ im Liter enthält. Die Lösung wird am Rückfluß 2 Stdn. dicht unter dem Siedepunkt gehalten. Das gebildete Kalomel wird im gewogenen Filtertiegel abfiltriert, erst mit heißem dest. Wasser, dann mit Alkohol und Äther gewaschen und schließlich bei $100{ }^{\circ} \mathrm{C}$ bis zur Gewichtskonstanz getrocknet.

Die 4 an der Probe Nr. 1 von Formica polyctena Först. ausgeführten Bestimmungen brachten folgendes Ergebnis:

[1951]; 234, 149 [1952]; Naturwissenschaften 47, 460 [1960].

5 R. Stumper, C. R. hebd. Séances Acad. Sci. 249, 1154 [1959] ; Mitt. Schweiz. Ent. Ges. 32, 374 [1959].

* Die Methode verdanken wir S. Sauerländer (unpubl.).

6 A. Scala, Gazz. chim. ital. 20, 393 [1890]; H. Franzen u. G. Greve, J. prakt. Chem. 80, 368 [1909]; O. Riesser, Hoppe-Seyler's Z. physiol. Chem. 96, 355 [1916]. 


\begin{tabular}{|c|c|c|c|c|c|c|}
\hline $\begin{array}{c}\text { Probe } \\
\text { Nr. }\end{array}$ & Art & Ort & Datum & $\begin{array}{c}\text { Ge- } \\
\text { wonnene } \\
\text { Rohgift- } \\
\text { menge } \\
\text { [mg] }\end{array}$ & $\begin{array}{c}\begin{array}{c}\text { Zahl } \\
\text { der }\end{array} \\
\text { benötigten } \\
\Varangle \varnothing \\
\Varangle q\end{array}$ & $\begin{array}{c}\text { Durch- } \\
\text { schnittl. } \\
\text { Körper- } \\
\text { gew. je } \varnothing \\
\text { [mg] }\end{array}$ \\
\hline $\begin{array}{r}1 \\
2 \\
3 \\
4 \\
5 \\
6 \\
7 \\
8 \\
9 \\
10\end{array}$ & $\begin{array}{l}F . \text { polyctena FöRst. } \\
F . \text { polyctena FöRsT. } \\
F . \text { polyctena FöRsT. } \\
\text { F. polyctena FöRst. } \\
\text { F. rufa L. } \\
\text { F. rufa L. } \\
\text { F. rufa L. } \\
\text { F. nigricans EM. } \\
\text { F. pratensis RETz.* } \\
\text { F. pratensis RETz.* }\end{array}$ & $\begin{array}{l}\text { Rengersbrunn } \\
\text { Rengersbrunn } \\
\text { Gramschatz } \\
\text { Lindelbach } \\
\text { Rengersbrunn } \\
\text { Gramschatz } \\
\text { Lindelbach } \\
\text { Rengersbrunn } \\
\text { Gramschatz } \\
\text { Lindelbach }\end{array}$ & $\begin{array}{r}20.2 .60 \\
7.8 .60 \\
6.8 .60 \\
5.8 .60 \\
7.8 .60 \\
6.8 .60 \\
5.8 .60 \\
7.8 .60 \\
\text { 6. 8. } 60 \\
\text { 5. } 8.60\end{array}$ & $\begin{array}{l}270,0 \\
312,0 \\
302,7 \\
196,9 \\
239,3 \\
228,3 \\
328,3 \\
318,3 \\
279,3 \\
263,8\end{array}$ & $\begin{array}{l}200 \\
255 \\
212 \\
265 \\
180 \\
210 \\
117 \\
185 \\
320 \\
244\end{array}$ & $\begin{array}{r}14,2 \\
9,5 \\
11,6 \\
5,3 \\
11,5 \\
10,5 \\
12,6 \\
13,7 \\
7,4 \\
10,4\end{array}$ \\
\hline
\end{tabular}

Tab. 1. Angaben über die verwendeten Ameisenarten der Gattung Formica, Datum der Probenentnahme, die gesammelten

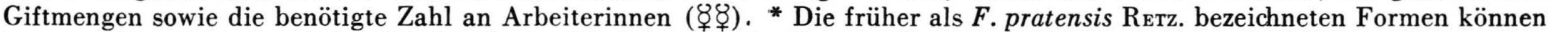
jetzt auf Grund taxonomischer Merkmale der Weibchen in die Formen F. nigricans Eм. und F. cordieri Bondr. differenziert werden. Da von den Nestern 9 und 10 noch keine Weibchen zugänglich waren, wird hier noch die Bezeichnung $F$. pratensis geführt.

$\begin{array}{lllll}\text { Einwaage [mg] } & 19,0 & 15,4 & 22,7 & 12,3 \\ \text { Ameisensäure [\%] } & 64,7 & 65,7 & 63,48 & 63,5\end{array}$

Die Ergebnisse beider Methoden stimmen also recht gut überein. Sie lassen den Schluß zu, daß außer Ameisensäure keine andere Säure in nennenswerter Menge vorliegt und ergaben, wie bereits erwähnt, daß auch die acidimetrische Methode hinreichend genaue Werte liefert.

In der Tab. 2 sind u. a. die für sämtliche 10 Proben gefundenen Mittelwerte für den Gehalt an
Ameisensäure aufgeführt. Überraschenderweise liegen alle Werte zwischen $61-65 \%$, es sind also weder deutliche Unterschiede zwischen den Arten, noch zwischen verschiedenen Standorten der gleichen Art bzw. zwischen Sommer- und Wintertieren festzustellen. Die von uns gefundenen Werte von durchschnittlich $63,5 \%$ liegen etwas höher als die von Oтто ${ }^{1}$ und STUMPER $^{5}$ angegebenen. Dies könnte vor allem auf die von den genannten Autoren verwendete andere Art der Giftgewinnung (man ließ die Ameisen auf Filtrierpapier spritzen) zurückzuführen sein.

\begin{tabular}{|c|c|c|c|c|c|c|c|c|c|c|}
\hline & \multicolumn{10}{|c|}{ Ameisenprobe Nr. } \\
\hline & 1 & 2 & 3 & 4 & 5 & 6 & 7 & 8 & 9 & 10 \\
\hline $\begin{array}{l}\text { Konzentration der } \\
\mathrm{HCOOH}[\%]\end{array}$ & 64,34 & 64,16 & 62,72 & 62,33 & 65,06 & 61,13 & 65,06 & 63,40 & 63,18 & 63,67 \\
\hline $\begin{array}{l}\text { Zur weiteren Analyse } \\
\text { verbleibendes Rohgift- } \\
\text { gewicht [mg] }\end{array}$ & 193,8 & 181,4 & 180,0 & 123,6 & 199,6 & 156,7 & 162,3 & 197,1 & 189,3 & 173,2 \\
\hline $\begin{array}{l}\text { Mit Aceton gefällte Stoffe } \\
\text { a) Gewicht [mg] }\end{array}$ & 0,10 & 1,10 & 0,91 & 1,01 & 0,76 & 0,10 & 0,82 & 1,18 & 0,95 & 0,94 \\
\hline b) $\%$ des Rohgiftgewichtes & 0,05 & 0,61 & 0,50 & 0,82 & 0,38 & 0,06 & 0,50 & 0,60 & 0,50 & 0,54 \\
\hline $\begin{array}{l}\text { AcetonlöslicheSubstanzen } \\
\text { a) Gewicht [mg] }\end{array}$ & 2,34 & 1,54 & 2,20 & 0,90 & 1,85 & 1,74 & 1,45 & 1,40 & 2,50 & 1,70 \\
\hline b) $\%$ des Rohgiftgewichtes & 1,22 & 0,85 & 1,22 & 0,73 & 0,93 & 1,11 & 0,89 & 0,71 & 1,32 & 0,99 \\
\hline $\begin{array}{l}\text { Gesamttrockensubstanzen } \\
\text { in } \% \text { des Rohgiftgewichtes }\end{array}$ & 1,27 & 1,46 & 1,72 & 1,55 & 1,31 & 1,17 & 1,39 & 1,31 & 1,82 & 1,53 \\
\hline $\begin{array}{l}\text { Im Rohgift gefundenes } \\
\mathrm{NH}_{3} \text { in \% des Gesamt- } \\
\text { trockengewichts }\end{array}$ & 5,82 & 0,03 & 0,10 & $\begin{array}{l}\text { nicht } \\
\text { be- } \\
\text { stimmt }\end{array}$ & 0,06 & 0,64 & $\begin{array}{c}\text { nicht } \\
\text { be- } \\
\text { stimmt }\end{array}$ & 0,06 & 0,15 & 0,05 \\
\hline
\end{tabular}

Tab. 2. Zahlenmäßiges Ergebnis der an den 10 Giftproben durchgeführten Bestimmungen der $\mathrm{HCOOH}$ und der 
Analyse der Begleitsubstanzen

Für alle Proben wurde nach Entnahme der zur HCOOH-Bestimmung benötigten Menge das verbleibende Rohgift weiter aufgearbeitet. Das Rohgift wurde mit dem etwa 10-fachen Volumen Aceton p.a. versetzt und der dabei ausgefällte Niederschlag abzentrifugiert. Diese Fällung wurde nochmals mit Aceton gewaschen, wieder abzentrifugiert, getrocknet und gewogen. In Tab. 2 ist das Gewicht der mit Aceton gefällten Stoffe (wohl vorzugsweise Proteine) sowie ihr Anteil (maximal $0,82 \%$ ) am Rohgiftgewicht aufgeführt.

Das Waschaceton wurde mit der acetonischen Lösung des Rohgiftes vereinigt, im Reagenzglas im Vakuum verdampft und $6 \mathrm{Stdn}$. lang im Hochvakuum bei $60^{\circ} \mathrm{C}$ über $\mathrm{P}_{2} \mathrm{O}_{5}$ getrocknet. Das Gewicht des Rückstandes, der die von Stumper vermutete Substanz von TerpenCharakter (soweit sie nicht bei der scharfen Trocknung im Hochvakuum flüchtig gegangen ist), daneben aber Eiweißbausteine, Salze u. a. enthalten dürfte, ist wiederum in Tab. 2 aufgeführt. Er beträgt $0,71-1,32 \%$ des Rohgiftgewichtes. Insgesamt liegt der Anteil an Trokkensubstanz bei den 10 untersuchten Proben zwischen 1,17 und 1,82 (im Mittel 1,45)\% des Rohgiftgewichtes, während nach STUmper ${ }^{5}$ 3,6-4,0\% Rückstand verblieben. Auch hier dürften sich die unterschiedlichen Ergebnisse aus der Verschiedenheit der Giftgewinnung, daneben aber auch der Möglichkeit von Verlusten bei der erwähnten scharfen Trocknung erklären lassen. Zudem könnte der terpenartige Stoff im Sekret der Nebendrüse (alkalische Drüse) enthalten sein, das wir im Gegensatz zu Stumper mit unserer Methode nicht erfassen.

Von zwei Proben, und zwar Nr. 1 ( $F$. polyctena im Überwinterungsstadium) und Nr. 3 (F. polyctena, Sommertiere), wurde der im Aceton lösliche Anteil auf Aminosäuren und Ammoniak untersucht. Um evtl. vorhandene Peptide zu hydrolysieren, wurde die Trockensubstanz mit $0,5 \mathrm{ml} 6-n$. $\mathrm{HCl}$ versetzt und $24 \mathrm{Stdn}$. bei $100{ }^{\circ} \mathrm{C}$ im zugeschmolzenen Röhrchen belassen. Nach dem Abdunsten der Salzsäure wurde in $4 \mathrm{ml}$ Puffer $p_{\mathrm{H}} 2,2$ gelöst und die Lösung einer säulenchromatographischen Aminosäuren-(und Ammoniak-) Analyse unterworfen ${ }^{7}$, deren Ergebnisse in Tab. 3 angegeben sind.

Auffällig ist, daß der Anteil an Aminosäuren und Ammoniak in der Winterameisenprobe Nr. 1 rund viermal so hoch ist $(19,85 \%)$ wie in der Sommerameisenprobe Nr. $3(4,83 \%)$. Da nur zwei Aminosäure-Analysen vorliegen, läßt sich für die Aminosäuren zwar nicht aussagen, daß dies mit der Winterruhe zusammenhängt; nachdem aber in 8 Proben das im Rohgift vorhandene $\mathrm{NH}_{3}$ bestimmt wurde (Tab. 2) und nur in der Winterprobe dessen

7 Beckman Spinco-Gerät zur vollautomatischen Trennung und quantitativen Bestimmung von Aminosäuren nach Spackman, Stein u. Moore, Anal. Chem. 30, 1190 [1958].

\begin{tabular}{|l|c|c|}
\hline \multirow{2}{*}{} & \multicolumn{2}{|c|}{$\begin{array}{c}\text { Gewichtsprozent, bezogen auf } \\
\text { Trockensubstanz }\end{array}$} \\
\cline { 2 - 3 } & Probe Nr. 1 & Probe Nr. 3 \\
\hline Alanin & 0,48 & 0,12 \\
Arginin & nicht bestimmt & Spur \\
Asparaginsäure & 1,46 & 0,35 \\
1/2 Cystin & Spur & Spur \\
Glutaminsäure & 1,70 & 0,69 \\
Glycin & 0,42 & 0,15 \\
Histidin & 0,18 & Spur \\
Isoleucin & 1,57 & 0,42 \\
Leucin & 2,78 & 1,04 \\
Lysin & 0,19 & Spur \\
Methionin & Spur & Spur \\
Phenylalanin & - & Spur \\
Prolin & 1,10 & 0,85 \\
Serin & 0,64 & 0,23 \\
Threonin & 0,58 & 0,08 \\
Tyrosin & 2,04 & 0,75 \\
Valin & 0,63 & Spur \\
Ammoniak & 6,08 & 0,15 \\
\hline Insgesamt & 19,85 & 4,83 \\
\hline
\end{tabular}

Tab. 3. Aminosäuren und Ammoniak aus der hydrolysierten acetonlöslichen Trockensubstanz der Ameisenproben Nr. 1 und Nr.3. Alle Zahlen bedeuten Gewichtsprozent, bezogen auf die Trockensubstanz (Probe $1=2,34 \mathrm{mg}$; Probe $3=$ $2,20 \mathrm{mg}$ ). Spur bedeutet, daß die betreffende Substanz in so geringer Menge auftrat, daß der zugehörige peak nicht auswertbar war.

Gehalt so auffällig hoch ist, darf doch eine Konzentration der Begleitsubstanzen des Ameisengiftes im Winter angenommen werden. Der hohe $\mathrm{NH}_{3}$-Gehalt in der Winterameisenprobe deutet eventuell darauf hin, daß die Abbauprodukte z. T. als Ammonsalze ausgeschieden werden. Nachdem eine Reihe von Hinweisen dafür vorliegt, daß die Drüsen z. B. Speicheldrüsen von Aphiden ${ }^{8}$ - bei Insekten exkretorische Funkticnen übernehmen, kann dies auch für die Giftdrüse von Formica angenommen werden.

In der acetonlöslichen Fraktion des Rohgiftes fanden sich auch folgende freie Aminosäuren in Spuren: Glutaminsäure, Glycin, Isoleucin, Leucin, Methionin und Tyrosin wurden öfters, Alanin, Asparaginsäure, Histidin, Prolin, Serin und Valin gelegentlich (davon Histidin nur einmal) gefunden. Ihre Menge war zu klein, um irgendwelche quantitative Auswertungen vornehmen oder Unterschiede zwischen den Proben herausarbeiten zu können.

8 W. Kloft u. P. Ehrhardt, Proc. Sympos. Radioisotopes and Radiation in Entomology of the I.A.A., Bombay/India (1960) i. pr.; K. E. Wohlfarth-Bottermann u. Moericke, Z. Zellforsch. mikroskop. Anatom. 52, 346 [1960]. 
Der mit Aceton aus dem Rohgift der Probe Nr. 1 ausgefällte Anteil wurde nach Lösen in wenigen Tropfen Wasser, in der Apparatur nach W i e l and und $\mathrm{Pfl}$ e i d e re r der Hochspannungs-Elektrophorese unterworfen. Bei niedrigem $p_{\mathrm{H}}\left(p_{\mathrm{H}} 1,9\right.$ und $\left.p_{\mathrm{H}} 3,5\right)$ konnte die Eiweißfraktion in 3 verschiedene Komponenten aufgetrennt werden, während beim $p_{\mathrm{H}} 6,5$ die aufgetragenen Proteine nicht wanderten, also am Start sitzen blieben. Ein mit Amidoschwarz $10 \mathrm{~b}$ angefärbter Parallelstreifen zeigte ein gleiches Ergebnis. Weitere Versuche waren wegen der geringen Menge des Materials nicht möglich.

$$
\text { Prüfung auf Phosphat }
$$

Durch den negativen Ausgang des an einer Probe (Nr. 1) durchgeführten Molybdänblau-Testes wurde festgestellt, daß im Gift kein Phosphat vorhanden ist.

Die Arbeiten wurden teils im Institut für Angewandte Zoologie (Vorstand Prof. Dr. K. Gösswald), teils im Chemischen Institut (Vorstand Prof. Dr. G. Fischer $\dagger$ ) der Universität Würzburg durchgeführt. Die Autoren danken den Vorständen der Institute und ihren Mitarbeitern für die Ermöglichung der Arbeiten sowie für ihre freundliche Unterstützung und stete Hilfe.

\title{
Die Schwärmperiodizität von Platynereis dumerilii im DD/LD-Belidhtungszyklus und nadh Augenausschaltung*
}

\author{
Von C. Hauenschild \\ Aus dem Zoologischen Institut der Universität Freiburg i. Br. \\ (Z. Naturforschg. 16 b, 753-756 [1961]; eingegangen am 12. Mai 1961)
}

\begin{abstract}
If periods with $\mathrm{DD}$ and $\mathrm{LD}$ alternate regularly the maximum of the swarming Heteronereis is nearly as distant from the beginning of DD as it is, under the alternation of LL- and LD-periods, from the beginning of LD. This supports the assumption that the position of the swarming maximum is determined by the moment at which the time of the daily illumination is shortened.

Blind Platynereis under the alternation of LL- and LD-periods have the same swarming periodicity as those with normal sight. Therefore the photoperiodic influences do not effect through the eyes.
\end{abstract}

I. Das Heteronereis-Schwärmen bei einem periodischen Wechsel von 1 Woche Dauerdunkel (DD) und 3 Wochen mit täglich je 16 Stdn. Licht (LD $16: 8$ )

Fragestellung: In den bisher veröffentlichten Versuchen über die Schwärmperiodizität von Platynereis dumerilii waren die Tiere einem rhythmischen Wechsel entweder zwischen einer Dauerlicht(LL) - und einer Normaltag(LD)-Periode bestimmter Länge oder zwischen 2 LD-Perioden mit unterschiedlicher Dauer der pro 24 Stdn. gegebenen Licht- und Dunkelzeiten (d. h. alternierend einer Kurz- und Langtagperiode) ausgesetzt ${ }^{1}$. Bei allen Variationen dieser Versuche folgte das Maximum des HeteronereisSchwärmens in einem vergleichbaren Abstand (16 bis 20 Tage) auf den Beginn der Kurztagperiode; dagegen war eine konstante Beziehung zwischen Schwärm-Maximum und Beginn der Langtag- bzw. LL-Periode nicht nachweisbar. Praktisch ließ sich also aus dem Zeitpunkt, an dem die tägliche Licht-

\footnotetext{
* Mit Unterstützung der D e u t s chen Forschungs. gemeins ch a f $\mathrm{t}$.
}

zeit verkürzt und die tägliche Dunkelzeit entsprechend verlängert wurde, die Lage des folgenden Schwärm-Maximums genau voraussagen. Dies brachte mich auf den Gedanken, daß die zum gehäuften Schwärmen führenden Metamorphose-Vorgänge im Rahmen eines 20 - 30-tägigen Belichtungszyklus durch die Verkürzung der täglichen Belichtungsdauer ausgelöst werden. Um die Allgemeingültigkeit dieser Vorstellung zu überprüfen, schien ein Versuch geeignet, in dem eine 3-wöchige LDPeriode (täglich 16 Stdn. Licht und 8 Stdn. Dunkelheit) mit einer 1-wöchigen DD (Dauerdunkel) Periode an Stelle der bisher üblichen LL(Dauerlicht)-Periode alternierte. Traf die obige Annahme in dieser allgemeinen Form zu, dann mußte in einem solchen Versuch das Schwärm-Maximum im üblichen Abstand auf den DD-Beginn und nicht, wie bei einem aus LL und LD bestehenden Belichtungszyklus, auf den LD-Beginn folgen.

Methode: Es wurden 2 Platynereis-Zuchten (insgesamt etwa 500 Individuen) im Alter von 3 Monaten in einen

\footnotetext{
1 C. Hauenschild, Cold Spring Harbor Sympos. quantitat. Biol. 25, 491 [1960].
} 